\title{
Video-assisted thoracoscopic surgery in lung cancer
}

\section{surgery}

\section{Editorial}

In 1910, the concept of thoracoscopy was first described by Jacobaeus. The following years, the thoracoscopy has become a common procedure for the lysis of pleural pulmonary adhesions caused is tuberculosis. The video-assisted thoracoscopic surgery (VATS) has been developed and continued to evolve in close connection with imaging technical advancements. VATS has been gaining popularity in the thoracic surgery not only in diagnosis, but also in the pneumothorax management. The oncologic aspects of VATS remain controversial. In a study on lung cancer surgery by Whitson et al, 5-yearsurvival rate in the VATS patients of $75 \%$ was compared to the lobectomy with thoracotomy. A "cold" halogen light source was introduced as another major step in enhancing the visualization of anatomical structures contributing to a major development in VATS since the blood in the operation field would absorb up to $50 \%$ of the light. The introduction of endostaplers was another milestone in VATS development. Because of emerging and evolution of VATS in the treatment of thoracic injuries, or resection of small pulmonary nodules, thus, many surgeons clearly believe that VATS is distinct from the traditional thoracoscopy in addition to its diagnostic roles. Generally, VATS is recognized as a modality dedicated to the management of early stage lung cancer (stage I and II) with no sign of lymph node invasion. Single-lung ventilation is mandatory for VATS lobectomy, so pulmonary function monitoring is important. VATS-segmentectomy is a safe option and provides comparable surgical results to VATS-lobectomy, particularly in stage IA NSCLC. Relative contraindications for VATS-lobectomy are listed as the following : 1.) tumor infiltration to the chest wall, 2) lymphadenopathy, 3) preoperative chemotherapy or radiation therapy, 4) bronchoscopic visible tumor, and 5) dense pleural adhesions (particularly for less experienced surgeons). Patients with more than $6 \mathrm{~cm}$. or T3 tumors are ineligible for VATS-lobectomy. The number of mediastinal or lymph node removed should be reported as an indicator of the quality of the surgical approach. Currently, robot-assisted surgery could be drawn attention as minimally invasive alternative to VATS. The robot-assisted surgery is promising not only for early stage-lung cancer, but also for more advanced and difficult cases, particularly, lymph-node dissection that seems to be easier and more radical than VATS lymphadenectomy.
Volume 6 Issue I - 2018

\author{
Attapon Cheepsattayakorn, ${ }^{1,2}$ Ruangrong \\ Cheepsattayakorn ${ }^{3}$ \\ 'IOth Zonal Tuberculosis and Chest Disease Center,Thailand \\ ${ }^{2}$ Department of Disease Control, Ministry of Public Health, \\ Thailand \\ ${ }^{3}$ Department of Pathology, Chiang Mai University, Thailand
}

Correspondence: Attapon Cheepsattayakorn, I0th Zonal Tuberculosis and Chest Disease Center, Department of Disease Control, Ministry of Public Health, Thailand, Tel 66-53-I40767, 66-53 276364, Fax 66-53-I40773, 66-53-273590, Email attapon1958@gmail.com

Received: December 09, 2017 | Published: February 19, 2018

\section{Conclusion}

In conclusion, although the controversial indications for VATS lobectomy, the evolution of VATS lobectomy is now driven by technological advancement and refinement of surgical instruments. The eligibility criteria for VATS lobectomy have been progressively extending to accommodate advanced stages of cancer and proper patient selection is considered of importance.

\section{Acknowledgements}

None.

\section{Conflict of interest}

The author declares no conflict of interest. 